\title{
Study of properties of jelly-fruit marmalade with herbal additives
}

\author{
Maya Artamonova ${ }^{1 *}$, Inna Piliugina ${ }^{2}$, and Olena Aksonova ${ }^{2}$ \\ ${ }^{1}$ Department of Technology of Bread, Confectionary, Pasta and Food Concentrates, Kharkiv State University of Food Technology \\ and Trade, Kharkiv, Ukraine \\ ${ }^{2}$ Department of Chemistry, Microbiology and Hygiene of Food, Kharkiv State University of Food Technology and Trade, Kharkiv, \\ Ukraine
}

\begin{abstract}
The results of studies of the properties of jelly-fruit marmalade with plant additives from Sudanese rose, rose hip and pumpkin during storage for 3 months are presented. It is shown that during the shelf life organoleptic, physicochemical quality indicators of marmalade with plant additives meet the requirements of current regulations, the color intensity is maintained. New products have a high content of $\beta$-carotene, anthocyanins, minerals, tannins and pectin.
\end{abstract}

\section{Introduction}

Confectionery is a large group of high-calorie foods that are in great demand among the population. In particular, there is an increase in production and demand for jelly products. This is due to their attractive appearance, pleasant taste, low price. However, the main significant disadvantage of confectionery is their low physiological value due to the almost complete absence of important biologically active substances, such as vitamins, dietary fiber, minerals and the like. Therefore, in today's market competition, it is important to improve existing technologies and develop new types of jelly products. This is possible through the development of innovative technologies, the creation of products of high nutritional and biological value, special purpose, as well as the use of new raw ingredients.

The search for new non-traditional types of raw materials for the production of jelly-fruit marmalade remains topical. Technologies for the manufacture of jelly products allow the introduction of plant supplements in various forms: in natural form, products of processing of plant raw materials, products of processing of nontraditional plant raw materials and their combinations.

Scientists are actively developing new technologies for jelly-fruit marmalade using plant raw materials or products of its processing. Thus, the technologies of fruit and jelly marmalade for health purposes with the use of rhubarb, elderberry and water-alcohol extracts of thyme, violet, oregano are known. The introduction of these additives allows to introduce into the finished product catechins, flavonols and anthocyanins. The finished product has good taste and appearance, increased nutritional value and pharmacological effect [1].The technology of jelly-fruit marmalade with alcohol extract of Dunaliella salina microalgae and concentrated apple juice has been developed. It is shown that new types of marmalade have increased antioxidant activity [2]. There is a method of obtaining jelly-fruit marmalade on agar and fructose with the addition of sea buckthorn juice. Replacement of apple puree with sea buckthorn juice in the marmalade formulation has significantly increased the antioxidant properties of products [3].

The technology of jelly-fruit marmalade using hydrated pectin, which is obtained from medicinal dandelion, has been developed. New technology allows to obtain products with increased nutritional value [4].Scientists have developed a technology of jelly-fruit marmalade on pectin with the replacement of granulated sugar with fructose and $30 \%$ of apple puree on pureed fruits chokeberry, which allowed to obtain a diabetic and functional product [5].The use of chokeberry puree in the recipe of jelly-fruit marmalade on agar allowed to obtain products with high content of anthocyanins [6].The expediency of using the pulp of the fruits of wild garlic in the production of jelly-fruit marmalade on agar of high nutritional value of functional orientation is proved [7].The possibility of introducing beet juice, sea buckthorn and cranberry puree into the recipe of jellyfruit marmalade on gelatin to increase its nutritional value has been established, namely the introduction of bioflavonoids and betaine into the composition of products[8].The use of semi-finished products from Jerusalem artichoke in the technology of fruit-jelly marmalade was proposed, which made it possible to increase the nutritional value of products and reduce the calorie content [9].

A number of publications are devoted to the use in the technology of jelly-fruit marmalade on pectin plant additives obtained by cryogenic technologies cryopowders and cryopaste [10-16].The use of cryopowders from grapes, rose hips, sea buckthorn and 
cryopaste from quince, apples, carrots, pumpkins, grapes in the production of jelly-fruit-marmalade to increase its nutritional value, complete exclusion from the prescription composition of synthetic dyes and flavors [10-12]. In addition, the introduction of these additives to the prescription composition of marmalade helped to increase the antioxidant properties of products that are stable during the guaranteed shelf life.[13, 14].It is shown that new types of jelly-fruit marmalade on pectin had high quality indicators during storage $[15,16]$.

In works $[17,18]$ the expediency of using in the technology of jelly-fruit marmalade on gelatin cryopaste and cryopowder from grapes is shown. The innovative solution allows to obtain high quality products with maximum preservation of biologically active substances and reduced consumption of apple puree by $10-15 \%$ and citric acid by $20-25 \%$, as well as the complete exclusion of dyes and flavors.

However, the task of increasing the nutritional value of jelly-fruit marmalade on gelatin through the use of plant supplements from Sudanese rose, rose hip and pumpkin in these works was not solved.

The authors of this publication are devoted to the solution of the problem described above. New types of jelly-fruit marmalade based on gelatin with herbal additives have been developed, namely Sudanese rose powder, rosehip cryopowder and pumpkin cryopaste. Studies of the properties of new types of marmalade with plant additives during the shelf life were not performed. The study of organoleptic and physicochemical properties of new products are necessary to justify their storage conditions and type of packaging.

Thus, to solve the problem of maintaining over time the quality, color fastness of new types of jelly-fruit marmalade on gelatin with plant additives from Sudan rose, rose hip and pumpkin, it is important to timely investigate their properties during storage.

\section{Experimental}

The purpose of this work was to study the organoleptic and physicochemical properties, chemical composition of jelly-fruit marmalade on gelatin with plant additives during storage, justification of optimal storage conditions for products and type of packaging.

Object of research - organoleptic, physicochemical properties and chemical composition of the product during its storage for 3 months. Subject of research jelly-fruit marmalade on gelatin with plant additives. Fruit jelly differed in the type of herbal additive Sudanese rose powder; cryopowder from rose hips and cryopaste from pumpkin (Fig. 1).

Marmalade made on gelatin according to traditional recipes was used as a control one.

Plant additives obtained by low-temperature technologies, due to fine grinding are a concentrate of biologically active substances. They contain a significant amount of low- and high-molecular phenolic compounds, dietary fiber, vitamins, glycosides, organic acids, macroand micronutrients and have antioxidant, immunomodulatory properties, as well as high coloring ability, good taste and aromatic characteristics.

The marmalade was stored at a temperature of $18 \pm 2$ ${ }^{\circ} \mathrm{C}$, relative humidity $75-80 \%$, without access to light for three months, packed in a plastic wrap and a cardboard box. Control points during the experiment were the $1 \mathrm{st}$, 2nd, 3rd months.

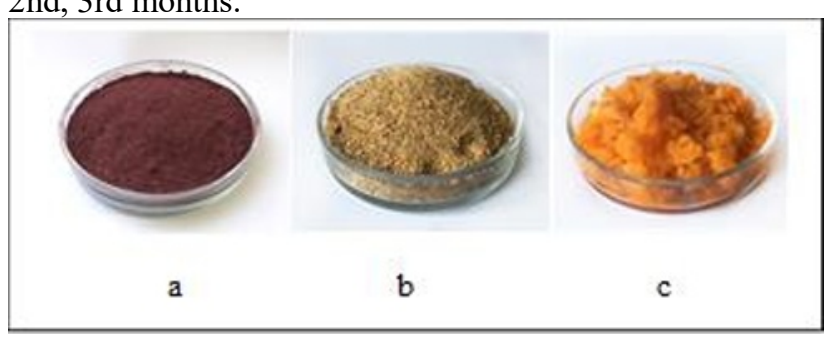

Fig. 1. Plant additives of: $\mathrm{a}$-Sudanese rose; $\mathrm{b}$ - rose hip; $\mathrm{c}-$ pumpkin.

Quantitative characterization of the quality of marmalade samples and its study during the shelf life was carried out based on the main physicochemical parameters, namely mass fraction of moisture, mass fraction of reducing substances, total acidity. These indicators were determined using standard methods [19, 20].

Color stability and color intensity of products with powder from Sudanese rose at certain time intervals were determined by spectrophotometric method. To do this, the optical density of the marmalade solution was determined on an SF-46 spectrophotometer at a wavelength of $510 \mathrm{~nm}$, using cuvettes with a layer thickness of 10-2 m. The change in optical density was expressed as a percentage, while the initial optical density was taken as $100 \%$.

The content of $\beta$-carotene was determined by colorimetric method. The method is based on the ability of $\beta$-carotene to dissolve in petroleum ether, as a result of which the solution turns yellow. The color intensity of the solution is proportional to the $\beta$-carotene content in it. Determination of the content of anthocyanin substances was carried out by the method of $\mathrm{pH}$ differential spectrophotometry. The content of pectin substances in new types of jelly-fruit marmalade was determined by the calcium pectate method, which is based on the precipitation of pectic acids in the form of calcium salts. The method of determination of tannins was based on their easy oxidation by potassium tetraoxomanganate in an acidic environment in the presence of indigosulfonic acid.

\section{Results and discussion}

Organoleptic and physicochemical quality indicators of jelly-fruit marmalade on gelatin with plant additives are given in tables 1,2 .

It was found that at the end of the shelf life, the visual perception of the color of new types of marmalade remained unchanged, the taste, appearance and consistency of the samples changed somewhat. Products decrease in volume, the consistency becomes gummier, 
the sour taste becomes more pronounced, which is confirmed by the data of physical and chemical studies.

It is proved that the color intensity of jelly-fruit marmalade with Sudanese rose powder after 3 months of storage was $100 \%$. Thus, the color of the new type of marmalade remains stable during the guaranteed shelf life.

The study of physicochemical parameters during storage for three months showed that at the end of the storage period the moisture content in new types of marmalade decreases to $11.9-13.4 \%$; the total acidity is 20.4-22.0 degrees; the content of reducing substances is $4.8-5.1 \%$.

The change in properties during storage is explained by a whole complex of physicochemical and microbiological processes. The transformations are associated with the hydrolysis of the carbohydrate component, redox reactions, condensation, and polymerization. However, all indicators were within the limits corresponding to the requirements of regulatory documents for this type of product.

The nutritional value of the developed types of marmalade was assessed. In jelly-fruit marmalade on gelatin with the addition of these plant additives, the content of minerals significantly increases (Table 3 ).

It is noted that the new types of jelly-fruit marmalade on gelatin in comparison with the sample made according to the traditional formulation, contain 1.2-2.3 times more phosphorus, 1.2-1.7 times more iron, 1.1-1.6 times more calcium. The content of potassium and magnesium also increases in the products. In general, with the introduction of herbal supplements, the mineral content in marmalade increases by 1.1-1.3 times.
It is determined that new types of marmalade with plant additives are characterized by a higher content of biologically active substances compared to jelly-fruit marmalade made according to traditional formulation (Table 4).

The $\beta$-carotene (mg / $100 \mathrm{~g}$ ) was found in freshly prepared marmalade: control contains $(1.30 \pm 0.04)$; marmalade with Sudanese rose powder - $(2.25 \pm 0.09)$; marmalade with rosehip cryopowder and pumpkin cryopaste - $(2.77 \pm 0.25)$. That is, the consumption of 100 $\mathrm{g}$ of freshly made marmalade provides $45 . .55 \%$ of $\beta$ carotene from the daily value of an adult.

After a month of storage, the content of $\beta$-carotene in the marmalade with pumpkin cryopaste and rosehip cryopowder was $0.15 \pm 0.03 \mathrm{mg}$ per $100 \mathrm{~g}$ of product. In jujube with powder from the Sudanese rose, $\beta$-carotene was destroyed, which is explained by its instability in an acidic condition and upon contact with atmospheric oxygen.

According to the results obtained (Table 4), the content of anthocyanins, pectin and tannins at the end of the shelf life remained almost unchanged. So, the possibility of storing jelly-fruit marmalade on gelatin with plant additives from Sudanese rose, rose hips and pumpkin has been proven during the guaranteed shelf life of 3 months, provided that it is packed in plastic wrap and a cardboard box.

It has been established that the consumption of $100 \mathrm{~g}$ of new types of marmalade will satisfy the daily need of healthy residents of Ukraine from 18 years of age and older in pectin substances by 3.7-5.7\%. Consuming $50 \mathrm{~g}$ of Sudanese rose marmalade will satisfy the daily need for anthocyanins by $120 \%$.

Table 1. Organoleptic quality indicators of freshly made jelly-fruit marmalade on gelatine with plant additiveseriment.

\begin{tabular}{|c|c|c|c|}
\hline \multirow{2}{*}{ Indicators } & \multicolumn{3}{|c|}{ Characteristics of jelly-fruit marmalade on gelatin } \\
\cline { 2 - 4 } & control & $\begin{array}{c}\text { with Sudanese rose } \\
\text { cryopowder }\end{array}$ & $\begin{array}{c}\text { with rose hips cryopowder and } \\
\text { pumpkin cryopaste }\end{array}$ \\
\hline Form & \multicolumn{3}{|c|}{ Correct, with a clear contour, without deformation } \\
\hline Surface & Yellow & Smooth, sprinkled with sugar \\
\hline Color & $\begin{array}{c}\text { Dark pink } \\
\text { Pleasant, without } \\
\text { Toreign taste and smell }\end{array}$ & $\begin{array}{c}\text { Pleasant, with a slight smack } \\
\text { of Sudanese rose without any } \\
\text { foreign taste and smell }\end{array}$ & $\begin{array}{c}\text { Pleasant, with a taste of pumpkin and } \\
\text { rose hips without foreign taste and } \\
\text { smell }\end{array}$ \\
\hline Consistence & \multicolumn{3}{|c|}{ Jellylike } \\
\hline
\end{tabular}

Table 2. Physicochemical indicators of the quality of jelly-fruit marmalade on gelatin with plant additives

\begin{tabular}{|c|c|c|c|c|}
\hline Sample of marmalade & $\begin{array}{l}\text { Shelf } \\
\text { life, } \\
\text { months }\end{array}$ & $\begin{array}{l}\text { Mass fraction } \\
\text { of moisture, \% }\end{array}$ & $\begin{array}{l}\text { Total acidity, } \\
\text { degrees }\end{array}$ & $\begin{array}{l}\text { Mass fraction of reducing } \\
\text { substances, } \%\end{array}$ \\
\hline \multirow{2}{*}{ Control } & 0 & $23.0 \pm 1.0$ & $11.6 \pm 0.9$ & $12.3 \pm 0.4$ \\
\hline & 3 & $12.9 \pm 0.9$ & $16.2 \pm 0.7$ & $4.2 \pm 0.7$ \\
\hline \multirow{2}{*}{ Marmalade with Sudanese rose cryopowder } & 0 & $22.2 \pm 1.2$ & $16.4 \pm 0.5$ & $14.3 \pm 0.4$ \\
\hline & 3 & $13.4 \pm 0.9$ & $22.0 \pm 0.3$ & $5.1 \pm 0.7$ \\
\hline \multirow{2}{*}{$\begin{array}{l}\text { Marmalade with rose hips cryopowder and } \\
\text { pumpkin cryopaste }\end{array}$} & 0 & $22.5 \pm 1.2$ & $13.2 \pm 0.5$ & $13.6 \pm 0.6$ \\
\hline & 3 & $11.9 \pm 0.8$ & $20.4 \pm 0.4$ & $4.8 \pm 0.8$ \\
\hline \multicolumn{2}{|c|}{ According to normative documents (DSTU 4333: 2004) } & $\begin{array}{l}\text { not more than } \\
24\end{array}$ & $7.5-22.5$ & not more than 28 \\
\hline
\end{tabular}


Table 3. The content of minerals in the studied sample of innovative marmalade

\begin{tabular}{|c|c|c|c|c|}
\hline \multirow{2}{*}{ Mineral substance } & \multirow{2}{*}{$\begin{array}{c}\text { Daily intake for an } \\
\text { adult, } \mathrm{mg}\end{array}$} & \multicolumn{3}{|c|}{ Content in marmalade, mg / $100 \mathrm{~g}$} \\
\cline { 3 - 5 } & control & $\begin{array}{c}\text { with Sudanese rose } \\
\text { cryopowder }\end{array}$ & $\begin{array}{c}\text { with rose hips cryopowder and } \\
\text { pumpkin cryopaste }\end{array}$ \\
\hline $\mathrm{K}$ & 2000 & 94.1 & 96.7 & 110.6 \\
\hline $\mathrm{Ca}$ & 1000 & 99.54 & 159.24 & 105.67 \\
\hline $\mathrm{Mg}$ & 400 & 24.34 & 25.02 & 1.9 \\
\hline $\mathrm{Fe}$ & 15 & 1.55 & 2.56 & 8.98 \\
\hline $\mathrm{P}$ & 800 & 3.90 & 4.63 & 253.22 \\
\hline Total & - & 223.43 & 282.86 & \\
\hline
\end{tabular}

Table 4. Chemical composition of jelly-fruit marmalade on gelatine with plant additives

\begin{tabular}{|c|c|c|c|c|}
\hline \multirow{2}{*}{ Sample of marmalade } & $\begin{array}{c}\text { Shelf life, } \\
\text { months }\end{array}$ & Anthocyanins, mg / 100 g & $\begin{array}{c}\text { Pectic substances, mg / } \\
100 \mathrm{~g}\end{array}$ & Tannins (by tannin), mg / 100 g \\
\cline { 3 - 5 } & & - & $182 \pm 2$ & $324 \pm 9$ \\
\hline \multirow{2}{*}{ Control } & 3 & - & $188 \pm 2$ & $320 \pm 9$ \\
\cline { 2 - 5 } & 0 & $124.0 \pm 6.2$ & $227 \pm 6$ & $352 \pm 10$ \\
\hline $\begin{array}{c}\text { Marmalade with Sudanese rose } \\
\text { cryopowder }\end{array}$ & 3 & $121.0 \pm 6.0$ & $230 \pm 7$ & $350 \pm 10$ \\
\cline { 2 - 5 } & 0 & $1.2 \pm 0.1$ & $187 \pm 5$ & $330 \pm 9$ \\
\hline $\begin{array}{c}\text { Marmalade with rose hips } \\
\text { cryopowder and pumpkin } \\
\text { cryopaste }\end{array}$ & 3 & $1.1 \pm 0.1$ & $187 \pm 5$ & $330 \pm 9$ \\
\cline { 2 - 5 }
\end{tabular}

\section{Conclusion}

Thus, the results of studies of the properties of jelly-fruit marmalade with plant additives from Sudanese rose, rose hips and pumpkin during storage for 3 months showed that during the storage period the organoleptic, physicochemical indicators of the quality of new products comply with the requirements of the current regulatory documentation, intensity color is preserved. New products have an increased content of $\beta$-carotene, anthocyanins, mineral, tannins and pectin substances.

Based on the results of research on new products, formulations and technology were developed, draft regulatory documents in the form of technical specifications and technological instructions, and the safety of the technology was assessed using the HACCP system.

\section{References}

1. A. Bashta, T. Leshchynska, Scientific Works of NUFT, 53, 63-70 (2013)

2. E. Kuznetsova, Ya. Brindza, E. Klimova, A. Borovkov, I. Gudvilovich, Ya. Jondareva, E. Kuznetsova, Food Industry, 4, pp. 14-19 (2019)

3. G. Magomedov, I. Lobosova., S. Zhurakhova, Technique and technology of food production, 46, 5054 (2017)

4. N. Tiguntseva, S. Evstafyev, News of higher educational institutions. Food technology, 4, 36-39 (2015)

5. L. Lobosova, M. Magomedov, S. Zhurakhova, Bulletin of the Voronezh State University of Engineering Technology, 4, 256-260 (2016)
6. A. Tabarovich, E. Stepanova, V. Bakaytis, Food industry. Raw materials and additives, 7, 53-57 (2017)

7. S. Glazyrin, N. Tipsina, Bulletin KrasGAU, 10, 218220 (2014)

8. E. Pankova, S. Agafonova, Bulletin of Youth Science, 14, 1-6 (2018)

9. G. Magomedov [et al.], Confectionery production, 4, 38-39 (2011)

10. M. Artamonova, N. Shmatchenko, Scientific works of the Odessa National Academy of Food Technologies, 46, 177-180,

11. M. Artamonova, N. Shmatchenko, Baker, 6, 36-37 (2015)

12. M. Artamonova, I. Piliugina, N. Shmatchenko, Nutrition: innovative aspects of technology, energyefficient production, storage and marketing, pp. 229256 (2015)

13. M. Artamonova, I. Piliugina, N. Shmatchenko, S. Gubsky, Nutrition: innovative aspects of technology, energy-efficient production, storage and marketing, 117-142 (2015)

14. S. Gubsky, M. Artamonova, N. Shmatchenko, I. Piliugina, E. Aksenova, Eastern-European Journal of Enterprise Technologies. Technology and equipment of food production, 4, 43-50 (2016)

15. N. Shmatchenko, M. Artamonova, O. Aksonova, S. Oliinyk, Food Science and Technology, 12, 87-94 (2018)

16. M. Artamonova, N. Shmatchenko, Study of the influence of plant cryoadditives on the quality of jelly-fruit marmalade during storage in Innovative aspects of food and hotel industry equipment 
development in modern conditions: materials of the second international scientific-practical conference, 2017, KhSUFTT, Kharkiv,Ukraine, pp. 203-204

17. M. Artamonova, N. Shmatchenko, I. Piliugina, Technology of jelly-fruit marmalade with the use of cryoadditives from grapes in International scientific and practical conference Technical sciences: history, the present time, the future, EU experience, 27-28 December 2019, Wloclawek, Poland
18. M. Artamonova., I. Piliugina, N. Shmatchenko, Nutrition: trends in energy efficient production, storage and marketing, 210-232 (2020)

19. Y. Picó, Chemical Analysis of Food: Techniques and Applications (Academic Press,Elsevier, 2012)

20. S.S. Nielsen, Food Analysis (Springer, Science+Business Media, LLC,2010) 